\title{
RESISTÊNCIA DE GENÓTIPOS DE CAUPI AO CARUNCHO'
}

\author{
PAULO DIÓGENES BARRETO² e MARY ANN WEYNE QUINDERÉ 3
}

RESUMO - A utilização de resistência genética ao ataque de Callosobruchus maculatus (Fabr.) tem sido alvo de investigação científica, especialmente no que diz respeito à identificação de fontes de resistência. O presente trabalho objetivou incorporar, ao grupo de caracteres desejáveis para o cultivo de caupi (Vigna unguiculata (L.) Walp.), resistência genética ao caruncho (C. maculatus). Foram realizadas hibridações dos genótipos IT81D-1045 e IT81D-1064 (portadores de resistência ao inseto) com CNCx 252-1E/FB, CNCx 187-22D-1 e BR 1-Poty (capazes de transferir resistência a viroses, tolerância à seca, formação de grãos com padrão comercial, elevado potencial de produção e adaptabilidade a diferentes condições ambientais). Populações segregantes obtidas destes cruzamentos foram conduzidas pelo método SPD (descendência de uma única vagem), e na geração $\mathrm{F}_{5}$ foram realizadas seleções individuais. As linhagens obtidas foram avaliadas em conjunto com materiais de origens diferentes, utilizando-se parâmetros associados à infestação da praga. Foi constatado que os genótipos avaliados apresentaram variabilidade quanto à preferência à postura, número de insetos emergidos e número de sementes danificadas; as linhas EVx $37-15 \mathrm{E}$ e EVx $37-2 \mathrm{E}$ foram as que sofreram menor dano causado pelo caruncho; as variáveis número de ovos, número de insetos emergidos e número de sementes danificadas mostraram-se positiva e significativamente correlacionadas entre si; o grupo das linhagens que descendem de genitores resistentes apresenta valores significativamente inferiores aos obtidos pelas demais, 0 que indica que a resistência ao inseto se transmite geneticamente.

Termos para indexação: Vigna unguiculata, melhoramento, resistência genética.

\section{RESISTANCE OF COWPEA GENOTYPES TO THE CALLOSOBRUCHUS MACULATUS}

\begin{abstract}
The use of genetic resistance to the weevil (Callosobruchus maculatus (Fabr.)) has been investigated to identify sources of resistance. The main purpose of the present work was to incorporate the genetic resistance to this insect into cowpea plants (Vigna unguiculata (L.) Walp.) with other already desirable. Hybridization was carried out among the genotypes IT81D-1045 and IT81D-1064 (identified in International Institute of Tropical Agriculture (IITA), Nigeria, as resistant to the insect) and CNCx 252-1E/FB, CNCx 187-22D-1 and BR 1-Poty, all of them able to transfer resistance to viruses, drought tolerance, desirable commercial quality of grains, high production potential and adaptability to different environments. Segregating populations were obtained from these crossings and so driven by the SPD method (single pod descendent) with individual selections being made in the $\mathrm{F}_{5}$ generation. The obtained lineages were assessed together with materials of different origins, using associated parameters to the pest infestation. Results revealed that the genotypes showed differences concerned to the eggs number laid by, number of insects hatched and the number of damaged seeds; these variables were positive by and significantly correlated among them; the EVx $37-15 \mathrm{E}$ and EVx 37-2E lineages were the less damaged by the weevil; the lineages group that descends from the resistant parents presented values significantly inferior to those obtained by the others, indicating that the resistance to the insect is genetically transmitted.
\end{abstract}

Index terms: Vigna unguiculata, improvement, genetic resistance, weevil.

\footnotetext{
${ }^{1}$ Aceito para publicação em 14 de junho de 1999.

${ }^{2}$ Eng. Agrôn., M.Sc., Embrapa-Centro Nacional de Pesquisa de Agroindústria Tropical (CNPAT), Rua Dra. Sara Mesquita, 2270, Pici, CEP 60511-110 Fortaleza, CE. E-mail: diogenes@cnpat.embrapa.br

${ }^{3}$ Eng. Agrôn, M.Sc., Empresa de Pesquisa Agropecuária do Ceará (EPACE), Av. Rui Barbosa, 1246, Aldeota, CEP 60115-221 Fortaleza, CE.
}

\section{INTRODUÇÃO}

A importância atribuída ao caruncho, (Callosobruchus maculatus (Fabr.)), enquanto praga dos grãos armazenados do caupi (Vigna unguiculata (L.) Walp.), deve-se a múltiplos aspectos e à intensidade dos danos. Iniciando a infestação, 
em certos casos, nas vagens, ainda no campo (Santos, 1971), o inseto é prejudicial, não somente pela redução do peso das sementes (Santos et al., 1978; Oliveira et al., 1984), mas também pela redução do valor nutritivo, pela queda do poder germinativo da semente, e por abrir caminho para a infecção por fungos (Santos \& Vieira, 1971); mas o dano maior é a alteração qualitativa do produto. A simples presença de ovos ou de insetos adultos, e até mesmo a constatação do odor característico que estes exalam, acarreta forte efeito restritivo sobre o consumo, com a conseqüente desvalorização comercial do produto (Bastos, 1973). O risco de infestação pelo inseto inibe as iniciativas de estocagem, tanto de grãos, no mercado atacadista, quanto de sementes, o que acentua a instabilidade de preços e restringe a possibilidade de incrementos de produtividade da cultura via difusão de cultivares melhoradas.

Como os meios eficazes de controle, em geral, através de silos metálicos herméticos, envolvem custos elevados e dificultam o manuseio de grandes estoques, a utilização de resistência genética ao ataque do caruncho tem sido alvo de investigação científica, especialmente no que diz respeito à identificação de fontes de resistência. Trabalhos como o de Santos (1976), Quinderé \& Barreto (1982), Fatunla \& Badaru (1983), Singh et al. (1985), Pessoa et al. (1993) e Chaves \& Vendramim (1995) demonstram ser viável o controle da praga por meio de cultivares geneticamente resistentes. Tal resistência se apresenta como um benefício direto ao produtor, pela redução de perdas pós-colheita, sem custos adicionais.

Este trabalho teve como objetivo incorporar, ao grupo de caracteres desejáveis para o cultivo de caupi, no Estado do Ceará, resistência genética ao caruncho (Callosobruchus maculatus (Fabr.)).

\section{MATERIAL E MÉTODOS}

O trabalho, em sua fase inicial, foi conduzido no Departamento de Pesquisa do Cariri (DPC/EPACE), em Barbalha, CE, a partir da definição de genitores. Entre 17 materiais introduzidos do IITA (International Institute of Tropical Agriculture), Nigéria, identificados como resis- tentes ao ataque do C. maculatus, dois, IT81D-1045 e IT81D-1064, foram eleitos, pela cor vermelha do grão (herança monogênica), resultando em menor dificuldade na condução das populações segregantes. Outros três parentais selecionados, originários do Programa Nacional de Melhoramento de Caupi, coordenado pela Embrapa-Centro Nacional de Pesquisa de Arroz e Feijão (CNPAF), foram as linhas CNCx 252-1E/FB e CNCx 187-22D-1 e a cultivar BR 1-Poty, portadoras de caracteres (resistência ao potyvirus, tegumento de cor marrom, alto potencial de produção e adaptabilidade) considerados de interesse relevante para o cultivo de caupi no Ceará. As populações segregantes que resultaram de cruzamentos entre os dois grupos de materiais (Tabela 1) foram conduzidas pelo método SPD (descendência de uma única vagem), e na geração $\mathrm{F}_{5}$ foram realizadas seleções individuais, optando-se por plantas que apresentavam o maior número de caracteres, previamente relacionados como de interesse para o agricultor do Estado.

Na segunda etapa do trabalho, realizada no Laboratório de Entomologia do Departamento de Pesquisa do Litoral (DPL/EPACE), em Maracanaú, CE, foram utilizados 102 materiais: 70 das linhas $\mathrm{F}_{5}$, obtidas dos cruzamentos para resistência ao C. maculatus: EVx 28-10E, EVx 28-12E, EVx 28-14E, EVx 28-15E, EVx 28-17E, EVx 28-18E, EVx 28-19E, EVx 28-1E, EVx 28-20E, EVx 28-21E, EVx 28-2E, EVx 28-3E, EVx 28-5E, EVx 28-6E, EVx 28-7E, EVx 28-8E, EVx 28-9E, EVx 30-1E, EVx 30-3E, EVx 30-4E, EVx 30-5E, EVx 30-6E, EVx 30-7E, EVx 31-10E, EVx 31-2E, EVx 31-3E, EVx 31-4E, EVx 31-5E, EVx 31-7E, EVx 31-9E, EVx 32-12E, EVx 32-14E, EVx 32-4E, EVx 32-5E, EVx 32-8E, EVx 32-9E, EVx 33-11E, EVx 33-12E, EVx 33-16E, EVx 33-4E, EVx 34-17E, EVx 34-18E,

TABELA 1. Cruzamentos realizados para inclusão de resistência genética ao caruncho Callosobruchus maculatus.

\begin{tabular}{ll}
\hline $\begin{array}{l}\text { Cruza- } \\
\text { mento }\end{array}$ & Origem (genitores) \\
\hline EVx 28 & IT81D-1045 x CNCx 252-1E/FB \\
EVx 29 & IT81D-1045 x BR 1-Poty \\
EVx 30 & IT81D-1045 x BR 1-Poty \\
EVx 31 & CNCx 187-22D-1 x IT81D-1045 \\
EVx 32 & IT81D-1045 x BR 1-Poty \\
EVx 33 & IT81D-1064 x BR 1-Poty \\
EVx 34 & IT81D-1045 x BR 1-Poty \\
EVx 35 & IT81D-1045 x BR 1-Poty \\
EVx 36 & IT81D-1045 x BR 1-Poty \\
EVx 37 & IT81D-1045 x BR 1-Poty \\
EVx 38 & IT81D-1064 x EVx 2 (CNCx 249-4F x BR 1-Poty) \\
\hline
\end{tabular}


EVx 34-1E, EVx 34-20E, EVx 34-2E, EVx 34-3E, EVx 35-6E, EVx 36-2E, EVx 37-10E, EVx 37-11E, EVx 37-12E, EVx 37-13E, EVx 37-14E, EVx 37-15E, EVx 37-18E, EVx 37-1E, EVx 37-20E, EVx 37-21E, EVx 37-22E, EVx 37-24E, EVx 37-2E, EVx 37-4E, EVx 37-5E, EVx 37-7E, EVx 37-8E, EVx 38-1E, EVx 38-2E, EVx 38-3E, EVx 38-4E, EVx 38-5E e EVx 38-6E; 26 seleções individuais da cultivar EPACE 10: EP10-S0/001, EP10-S0/015, EP10-S0/028, EP10-S0/089, EP10-S0/225, EP10-S0/289, EP10-S1/020, EP10-S1/021, EP10-S1/022, EP10-S1/059, EP10-S1/060, EP10-S1/201, EP10-S1/206, EP10-S1/240, EP10-S1/376, EP10-S2/260, EP10-S2/267, EP10-S2/272, EP10-S2/273, EP10-S2/274, EP10-S2/287, EP10-S2/308, EP10-S2/355, EP10-S2/363, EP10-S2/374 e EP10-SO/122, e seis cultivares comerciais: BR 1-Poty, BR 10-Piauí, EPACE 1, EPACE 10, EPACE 11 e EPACE V-96.

As observações sobre postura (número de ovos por amostra), número de insetos emergidos e número de grãos danificados, foram realizadas à temperatura ambiente média de $28,2^{\circ} \mathrm{C}$ e umidade relativa média de $69,1 \%$. Adotouse o delineamento experimental inteiramente casualizado, com quatro repetições. Amostras de 30 grãos de cada material foram colocadas em recipientes de plástico transparente e rígido, volume de $50 \mathrm{~mL}$, contendo uma lixa. Em cada recipiente, foram colocados dois casais de caruncho com um dia de idade, e transferidos, a cada 24 horas, para novas amostras de 30 grãos do mesmo material, repetindose esse procedimento até 72 horas (três dias de idade dos insetos). A contagem dos ovos foi realizada seis dias após a retirada dos insetos. Os recipientes foram mantidos em prateleiras, observados diariamente, e assim que os adultos emergiam, eram contados e retirados. Os dados foram registrados em cada um dos três dias de postura.

Os dados transformados para $\log (\mathrm{x}+1)$ foram analisados quanto à variância, pelo teste $\mathrm{F}$, e as médias, comparadas pelo teste de Tukey (5\%). Além disso, foram realizadas análises de correlação e de regressão entre as variáveis, e comparados, por contraste, os grupos de genótipos, de acordo com a origem.

\section{RESULTADOS E DISCUSSÃO}

As análises de variância revelaram significância no que se refere a oviposição, insetos emergidos e sementes danificadas.

A linhagem EVx 31-3E foi a menos procurada para oviposição, seguida da EVx 37-13E, EVx 31-7E, EVx 31-9E e EVx 37-15E, enquanto EP10-S2/267,EP10-S1/201,EP10-S2/272,EP10-S2/355 e EPACE 10 foram as mais procuradas (Tabela 2). A variação entre genótipos, quanto à preferência para postura, também foi verificada por Santos (1971, 1976), Chaves \& Vendramim (1995). De maneira geral, os materiais em que um dos progenitores era resistente à praga C. maculatus (linhagens EVx's) foram os que apresentaram menor postura, o que confirma resultados obtidos por Redden et al. (1983), citados por Araújo et al. (1988). Em relação à oviposição por dia de exposição, constatou-se que no primeiro dia o número de ovos foi significativamente maior (Tabela 2), resultado coerente com o de Santos (1971). Entre o segundo e o terceiro dia de postura, os valores não diferiram entre si.

Com relação ao número de adultos emergidos, destacam-se as linhas EVx 37-15E, EVx 37-2E, EP10-S0-122, EVx 37-13E, EP10-S1-059 e EVx 31-3E, enquanto EP10-S2-272, EP10-S1-201, EPACE 10, EP10-S2-355, EP10-S1-206 e EP10-S2-267 foram as que apresentaram maior quantidade de insetos emergidos (Tabela 2). Verifica-se correlação positiva entre a preferência para oviposição e o número de insetos emergidos. Relacionando-se adultos emergidos com o dia de postura, observa-se que o maior número emergiu dos ovos do primeiro e terceiro dia de postura. A postura do segundo dia deu origem a menos insetos (Tabela 2). Estes resultados diferem dos obtidos por Santos (1971), que constatou maior número de insetos emergidos das posturas do segundo e terceiro dia.

Por outro lado, nem sempre as cultivares mais ovipositadas são suscetíveis, pois outros fatores podem impedir o desenvolvimento larval do inseto, e um genótipo muito ovipositado pode ainda se revelar resistente, o que não aconteceu com a EPACE 10 e EPACE 11, já que foram suscetíveis no tocante aos dois parâmetros (Tabela 2).

Como semente danificada depende de inseto emergido, ressalta-se que os materiais que evidenciaram menor número de sementes furadas foram os que mostraram também menor número de insetos emergidos (EVx 37-15E, EVx 37-2E, EP10-S0/122 e EVx 37-13E) (Tabela 2).

O contraste entre as médias das linhagens cujos progenitores eram portadores de resistência ao caruncho (linhagens EVx's) com os demais genótipos 
TABELA 2. Número médio de ovos, insetos emergidos e sementes danificadas, na progênie de dois casais de Callosobruchus maculatus, criados em sementes de cultivares e linhagens de caupi (Vigna unguiculata) ${ }^{1}$.

\begin{tabular}{|c|c|c|c|}
\hline Cultivar/linhagem & $\begin{array}{l}\text { Número } \\
\text { de ovos }\end{array}$ & $\begin{array}{c}\text { Número } \\
\text { de insetos emergidos }\end{array}$ & $\begin{array}{c}\text { Número } \\
\text { de sementes danificadas }\end{array}$ \\
\hline EVx $37-15 E$ & 1,06 pqrst & $0,44 \mathrm{t}$ & $0,42 \mathrm{~m}$ \\
\hline EVx $37-2 E$ & 1,42 defghijklmnopqrst & $0,45 \mathrm{st}$ & $0,45 \mathrm{~m}$ \\
\hline $\mathrm{EP} 10-\mathrm{SO} / 122$ & 1,54 abcdefghijklmnopqrst & $0,54 \mathrm{rst}$ & $0,53 \mathrm{~lm}$ \\
\hline EVx $37-13 E$ & $0,91 \mathrm{st}$ & $0,58 \mathrm{rst}$ & $0,53 \mathrm{~lm}$ \\
\hline EVx $31-3 E$ & $0,89 \mathrm{t}$ & 0,59 pqrst & $0,56 \mathrm{klm}$ \\
\hline EP10-S1/059 & 1,54 abcdefghijklmnopqrst & 0,58 qrst & $0,58 \mathrm{jklm}$ \\
\hline EVx $32-5 E$ & 1,14 nopqrst & 0,63 pqrst & $0,61 \mathrm{ijklm}$ \\
\hline EVx $38-2 E$ & 1,35 hijklmnopqrst & 0,63 pqrst & $0,62 \mathrm{ijklm}$ \\
\hline EVx 37-24E & 1,34 hijklmnopqrst & 0,68 nopqrst & $0,63 \mathrm{ijklm}$ \\
\hline $\mathrm{EV} \times 37-4 \mathrm{E}$ & 1,34 hijklmnopqrst & 0,65 opqrst & 0,63 ijklm \\
\hline EVx $33-12 E$ & 1,42 defghijklmnopqrst & 0,66 opqrst & 0,64 hijklm \\
\hline $\mathrm{EVx} 28-15 \mathrm{E}$ & 1,31 jklmnopqrst & 0,67 opqrst & 0,65 hijklm \\
\hline EVx 33-4E & 1,23 lmnopqrst & 0,65 opqrst & 0,65 hijklm \\
\hline $\mathrm{EV} \times 37-12 \mathrm{E}$ & 1,39 efghijklmnopqrst & 0,69 nopqrst & 0,66 hijklm \\
\hline EVx $31-7 E$ & $0,98 \mathrm{rst}$ & 0,71 lmnopqrst & 0,67 ghijklm \\
\hline $\mathrm{EVx} 31-9 \mathrm{E}$ & 1,00 qrst & 0,71 lmnopqrst & 0,67 ghijklm \\
\hline $\mathrm{EV} \times 30-4 \mathrm{E}$ & 1,42 defghijklmnopqrst & 0,72 lmnopqrst & 0,68 fghijklm \\
\hline EVx $37-11 E$ & 1,41 defghijklmnopqrst & 0,69 mnopqrst & 0,68 ghijklm \\
\hline EP10-S2/287 & 1,33 hijklmnopqrst & $0,80 \mathrm{jklmnopqrst}$ & 0,70 defghijklm \\
\hline EVx 34-18E & 1,41 defghijklmnopqrst & 0,72 lmnopqrst & 0,70 defghijklm \\
\hline $\mathrm{EV} \times 35-6 \mathrm{E}$ & 1,37 fghijklmnopqrst & $0,78 \mathrm{jklmnopqrst}$ & 0,70 efghijklm \\
\hline$E V x 32-12 E$ & 1,40 defghijklmnopqrst & 0,73 lmnopqrst & 0,73 defghijklm \\
\hline EVx $37-5 E$ & 1,37 ghijklmnopqrst & $0,80 \mathrm{jklmnopqrst}$ & 0,74 defghijklm \\
\hline EVx $33-11 E$ & 1,40 defghijklmnopqrst & 0,76 klmnopqrst & 0,75 defghijklm \\
\hline $\mathrm{EV} \times 28-8 \mathrm{E}$ & 1,12 opqrst & $0,81 \mathrm{jklmnopqrst}$ & 0,77 cdefghijklm \\
\hline EVx $37-18 E$ & 1,42 defghijklmnopqrst & $0,79 \mathrm{jklmnopqrst}$ & 0,77 cdefghijklm \\
\hline EVx $38-1 E$ & 1,32 ijklmnopqrst & 0,83 jklmnopqrst & 0,77 cdefghijklm \\
\hline BR10-Piauí & 1,38 fghijklmnopqrst & 0,80 jklmnopqrst & 0,78 bcdefghijklm \\
\hline EVx 34-17E & 1,57 abcdefghijklmnopqrst & 0,84 jklmnopqrst & 0,78 bcdefghijklm \\
\hline $\mathrm{EV} \times 37-10 \mathrm{E}$ & 1,60 abcdefghijklmnopqrs & 0,88 ijklmnopqrst & 0,78 abcdefghijklm \\
\hline $\mathrm{EV} \times 31-10 \mathrm{E}$ & 1,26 klmnopqrst & 0,80 jklmnopqrst & 0,79 abcdefghijklm \\
\hline EVx 32-9E & 1,57 abcdefghijklmnopqrst & 0,79 jklmnopqrst & 0,79 abcdefghijklm \\
\hline $\mathrm{EV} \times 37-14 \mathrm{E}$ & 1,43 defghijklmnopqrst & $0,83 \mathrm{jklmnopqrst}$ & 0,80 abcdefghijklm \\
\hline EVx $37-8 E$ & 1,47 cdefghijklmnopqrst & 0,90 hijklmnopqrst & 0,80 abcdefghijklm \\
\hline $\mathrm{EVx} 38-3 \mathrm{E}$ & 1,71 bcdefghijklmnop & 0,81 jklmnopqrst & 0,81 abcdefghijklm \\
\hline $\mathrm{EVx} 28-14 \mathrm{E}$ & 1,34 hijklmnopqrst & 0,89 ijklmnopqrst & 0,82 abcdefghijklm \\
\hline $\mathrm{EVx} 28-20 \mathrm{E}$ & 1,33 hijklmnopqrst & 0,90 hijklmnopqrst & 0,82 abcdefghijklm \\
\hline EVx 28-9E & 1,36 hijklmnopqrst & 0,84 jklmnopqrst & 0,82 abcdefghijklm \\
\hline $\mathrm{EVx} 32-4 \mathrm{E}$ & 1,45 defghijklmnopqrst & 0,88 ijklmnopqrst & 0,82 abcdefghijklm \\
\hline EVx $28-18 E$ & 1,25 klmnopqrst & 0,89 ijklmnopqrst & 0,83 abcdefghijklm \\
\hline $\mathrm{EP} 10-\mathrm{S} 0 / 225$ & 1,69 abcdefghijklmnopq & 0,92 ghijklmnopqrst & 0,86 abcdefghijklm \\
\hline EP10-S2/374 & $1,28 \mathrm{jklmnopqrst}$ & 1,20 abcdefghijklmnopqrst & 0,86 abcdefghijklm \\
\hline $\mathrm{EVx} 28-1 \mathrm{E}$ & 1,32 ijklmnopqrst & 0,88 ijklmnopqrst & 0,86 abcdefghijklm \\
\hline EVx $36-2 E$ & 1,58 abcdefghijklmnopqrst & 0,94 ghijklmnopqrst & 0,86 abcdefghijklm \\
\hline $\mathrm{EV} x 37-7 \mathrm{E}$ & 1,53 abcdefghijklmnopqrst & 0,92 ghijklmnopqrst & 0,87 abcdefghijklm \\
\hline EP10-S0/089 & 1,56 abcdefghijklmnopqrst & 0,89 ijklmnopqrst & 0,88 abcdefghijklm \\
\hline EVx 34-1E & 1,59 abcdefghijklmnopqrs & 0,98 efghijklmnopqrst & 0,89 abcdefghijklm \\
\hline EVx $30-7 E$ & 1,34 hijklmnopqrst & 0,92 ghijklmnopqrst & 0,90 abcdefghijklm \\
\hline $\mathrm{EVx} 28-5 \mathrm{E}$ & 1,22 mnopqrst & 0,99 defghijklmnopqrst & 0,93 abcdefghijklm \\
\hline $\mathrm{EVx} 28-7 \mathrm{E}$ & 1,38 fghijklmnopqrst & 0,97 efghijklmnopqrst & 0,93 abcdefghijklm \\
\hline EVx $33-16 E$ & 1,39 defghijklmnopqrst & 0,95 fghijklmnopqrst & 0,93 abcdefghijklm \\
\hline EVx 34-2E & 1,69 abcdefghijklmnop & 0,99 defghijklmnopqrst & 0,93 abcdefghijklm \\
\hline EVx $28-3 E$ & 1,42 defghijklmnopqrst & 1,00 defghijklmnopqrst & 0,94 abcdefghijklm \\
\hline
\end{tabular}


TABELA 2. Continuação.

\begin{tabular}{|c|c|c|c|}
\hline Cultivar/linhagem & $\begin{array}{l}\text { Número } \\
\text { de ovos }\end{array}$ & $\begin{array}{c}\text { Número } \\
\text { de insetos emergidos }\end{array}$ & $\begin{array}{c}\text { Número } \\
\text { de sementes danificadas }\end{array}$ \\
\hline $\mathrm{EVx} 37-22 \mathrm{E}$ & 1,81 abcdefghijklmn & 0,96 fghijklmnopqrst & 0,94 abcdefghijklm \\
\hline EVx $30-3 E$ & 1,54 abcdefghijklmnopqrst & 1,03 cdefghijklmnopqrst & 0,95 abcdefghijklm \\
\hline EVx $30-6 E$ & 1,40 defghijklmnopqrst & 0,98 efghijklmnopqrst & 0,95 abcdefghijklm \\
\hline EVx 34-20E & 1,55 abcdefghijklmnopqrst & 1,02 defghijklmnopqrst & 0,95 abcdefghijklm \\
\hline EVx $31-5 E$ & 1,48 abcdefghijklmnopqrst & 1,00 defghijklmnopqrst & 0,96 abcdefghijklm \\
\hline EVx $37-1 E$ & 1,47 cdefghijklmnopqrst & 0,99 defghijklmnopqrst & 0,96 abcdefghijklm \\
\hline EVx $38-4 E$ & 1,53 abcdefghijklmnopqrst & 0,99 defghijklmnopqrst & 0,96 abcdefghijklm \\
\hline $\mathrm{EV} x 28-6 \mathrm{E}$ & 1,39 efghijklmnopqrst & 1,04 cdefghijklmnopqrst & 0,97 abcdefghijklm \\
\hline$E V x 32-14 E$ & 1,58 abcdefghijklmnopqrst & 0,98 efghijklmnopqrst & 0,97 abcdefghijklm \\
\hline EPACE V-96 & 1,38 fghijklmnopqrst & 1,27 abcdefghijklmnopqrs & 0,99 abcdefghijklm \\
\hline $\mathrm{EP} 10-\mathrm{S} 2 / 363$ & 1,67 abcdefghijklmnopq & 1,13 abcdefghijklmnopqrst & 0,99 abcdefghijklm \\
\hline EPACE 1 & 1,56 abcdefghijklmnopqrst & 1,11 abcdefghijklmnopqrst & 1,00 abcdefghijklm \\
\hline $\mathrm{EVx} 28-12 \mathrm{E}$ & 1,45 defghijklmnopqrst & 1,06 cdefghijklmnopqrst & 1,00 abcdefghijklm \\
\hline EVx 31-2E & 1,34 hijklmnopqrst & 1,07 bcdefghijklmnopqrst & 1,01 abcdefghijklm \\
\hline $\mathrm{EVx} 28-2 \mathrm{E}$ & 1,44 defghijklmnopqrst & 1,07 abcdefghijklmnopqrst & 1,03 abcdefghijklm \\
\hline EVx $30-5 E$ & 1,44 defghijklmnopqrst & 1,11 abcdefghijklmnopqrst & 1,03 abcdefghijklm \\
\hline EVx $32-8 E$ & 1,44 defghijklmnopqrst & 0,86 jklmnopqrst & 1,03 abcdefghijklm \\
\hline $\mathrm{EV} x 28-10 \mathrm{E}$ & 1,57 abcdefghijklmnopqrst & 1,13 abcdefghijklmnopqrst & 1,04 abcdefghijklm \\
\hline $\mathrm{EVx} 28-21 \mathrm{E}$ & 1,58 abcdefghijklmnopqrst & 1,12 abcdefghijklmnopqrst & 1,06 abcdefghijklm \\
\hline EP10-S1/021 & 1,63 abcdefghijklmnopqr & 1,37 abcdefghijklmnopq & 1,07 abcdefghijklm \\
\hline EVx 28-17E & 1,50 abcdefghijklmnopqrst & 1,16 abcdefghijklmnopqrst & 1,07 abcdefghijklm \\
\hline EVx 34-3E & 1,66 abcdefghijklmnopqr & 1,19 abcdefghijklmnopqrst & 1,08 abcdefghijklm \\
\hline EP10-S2/274 & 1,79 abcdefghijklmno & 1,42 abcdefghijklmnop & 1,10 abcdefghijklm \\
\hline EVx 37-21E & 1,56 abcdefghijklmnopqrst & 1,14 abcdefghijklmnopqrst & 1,10 abcdefghijklm \\
\hline EVx 28-19E & 1,47 abcdefghijklmnopqrst & 1,20 abcdefghijklmnopqrst & 1,14 abcdefghijkl \\
\hline $\mathrm{EP} 10-\mathrm{S} 0 / 289$ & 1,92 abcdefghijkl & 1,28 abcdefghijklmnopqrs & 1,15 abcdefghijkl \\
\hline EP10-S2/260 & 1,79 abcdefghijklmno & 1,60 abcdefghij & 1,16 abcdefghijkl \\
\hline EVx $31-4 E$ & 1,44 defghijklmnopqrst & 1,22 abcdefghijklmnopqrst & 1,16 abcdefghijkl \\
\hline EP10-S2/308 & 1,84 abcdefghijklm & 1,51 abcdefghijklmn & 1,17 abcdefghijkl \\
\hline EVx 37-20E & 1,59 abcdefghijklmnopqrs & 1,19 abcdefghijklmnopqrst & 1,17 abcdefghijkl \\
\hline EVx $38-5 E$ & 1,51 abcdefghijklmnopqrst & 1,24 abcdefghijklmnopqrst & 1,17 abcdefghijkl \\
\hline EVx 30-1E & 1,57 abcdefghijklmnopqrst & 1,25 abcdefghijklmnopqrst & 1,18 abcdefghijkl \\
\hline EP10-S2/273 & 1,80 abcdefghijklmno & 1,54 abcdefghijkl & 1,23 abcdefghijk \\
\hline EVx $38-6 E$ & 1,51 abcdefghijklmnopqrst & 1,31 abcdefghijklmnopqr & 1,24 abcdefghij \\
\hline EPACE 11 & 1,96 abcdefghij & 1,57 abcdefghijk & 1,26 abcdefghij \\
\hline EP10-S0/015 & 1,97 abcdefghij & 1,56 abcdefghijk & 1,27 abcdefghi \\
\hline EP10-S1/022 & 2,01 abcdefgh & 1,72 abcdefgh & 1,27 abcdefghi \\
\hline EP10-S0/001 & 1,93 abcdefghijk & 1,52 abcdefghijklm & 1,28 abcdefghi \\
\hline EP10-S1/020 & 1,97 abcdefghij & 1,47 abcdefghijklmno & 1,28 abcdefghi \\
\hline EP10-S1/376 & 1,81 abcdefghijklm & 1,74 abcdefg & 1,32 abcdefgh \\
\hline EP10-S2/267 & 2,17 a & 1,79 abcde & 1,34 abcdefg \\
\hline EP10-S1/060 & 2,06 abcdef & 1,78 abcdef & 1,36 abcde \\
\hline EP10-S1/240 & 2,01 abcdefghi & 1,69 abcdefghi & 1,36 abcdef \\
\hline EP10-S0/028 & 2,05 abcdefg & 1,69 abcdefghi & 1,37 abcde \\
\hline EP10-S1/206 & 2,06 abcdefg & 1,79 abcde & 1,38 abcd \\
\hline EPACE 10 & 2,08 abcd & $1,89 \mathrm{ab}$ & $1,44 a b c$ \\
\hline EP10-S1/201 & $2,16 \mathrm{ab}$ & $1,85 \mathrm{abc}$ & $1,44 \mathrm{abc}$ \\
\hline EP10-S2/272 & $2,15 \mathrm{abc}$ & $1,89 \mathrm{a}$ & $1,46 \mathrm{ab}$ \\
\hline EP10-S2/355 & 2,08 abcde & $1,82 \mathrm{abcd}$ & 1,46 a \\
\hline \multicolumn{4}{|l|}{ Dia de exposição } \\
\hline $1^{\circ} \mathrm{dia}$ & $1,65 \mathrm{a}$ & $1,13 \mathrm{a}$ & $1,00 \mathrm{a}$ \\
\hline $2^{\circ} \mathrm{dia}$ & $1,47 \mathrm{~b}$ & $0,88 \mathrm{~b}$ & $0,80 \mathrm{~b}$ \\
\hline $3^{\circ} \mathrm{dia}$ & $1,47 \mathrm{~b}$ & $1,14 \mathrm{a}$ & $1,02 \mathrm{a}$ \\
\hline
\end{tabular}

Continua... 
TABELA 2. Continuação.

\begin{tabular}{|c|c|c|c|}
\hline Cultivar/linhagem & $\begin{array}{l}\text { Número } \\
\text { de ovos }\end{array}$ & $\begin{array}{c}\text { Número } \\
\text { de insetos emergidos }\end{array}$ & $\begin{array}{c}\text { Número } \\
\text { de sementes danificadas }\end{array}$ \\
\hline \multicolumn{4}{|l|}{ Contraste: $\mathrm{EVX} *$ demais linhagens } \\
\hline Demais & $1,81 \mathrm{a}$ & $1,41 \mathrm{a}$ & $1,13 \mathrm{a}$ \\
\hline EVx & $1,40 \mathrm{~b}$ & $0,89 \mathrm{~b}$ & $0,85 \mathrm{~b}$ \\
\hline$\sigma^{2}$ & 0,46 & 0,53 & 0,47 \\
\hline C.V. $(\%)$ & 25,53 & 44,46 & 40,81 \\
\hline $\mathrm{QME}^{-1 / 2}$ & 0,39 & 0,47 & 0,38 \\
\hline Média & 1,53 & 1,05 & 0,94 \\
\hline \multicolumn{4}{|l|}{ Estatísticas dos dados originais } \\
\hline \multicolumn{4}{|l|}{ Médias } \\
\hline Demais linhagens & 112,26 & 58,25 & 19,43 \\
\hline EVx & 31,24 & 11,53 & 9,10 \\
\hline \multicolumn{4}{|l|}{ Desvio padrão $(\sigma)$} \\
\hline Demais linhagens & 100,91 & 58,73 & 11,68 \\
\hline EVx & 23,30 & 13,76 & 7,48 \\
\hline F-Linhagem & $6,03 * *$ & $7,08 * *$ & $4,98 * *$ \\
\hline F-Dia de exposição & $27,45^{* *}$ & $39,86^{* *}$ & $39,38 * *$ \\
\hline F-Dia exp.(linha) & $0,64^{\mathrm{ns}}$ & $1,09^{\mathrm{ns}}$ & $1,14^{\mathrm{ns}}$ \\
\hline \multicolumn{4}{|l|}{ F-Contrastes } \\
\hline EVx*Demais linhagens & $432,12 * *$ & $285,86^{* *}$ & $127,43 * *$ \\
\hline $\mathrm{EVx}(\mathrm{EVx} *$ Demais linhagens $)$ & $3,78 * *$ & $1,91 * *$ & $2,38 * *$ \\
\hline Demais(EVx*Demais linhagens) & $8,54 * *$ & $7,84 * *$ & $4,91 * *$ \\
\hline
\end{tabular}

${ }^{1}$ Médias seguidas da mesma letra não diferem entre si pelo teste de Tukey a 5\% de probabilidade; médias transformadas em logaritmo. ** Significativo a $1 \%$ de probabilidade.

revela que tanto a oviposição, como o número de insetos emergidos e o número de sementes danificadas foram significativamente menores nos materiais do primeiro grupo (Tabela 2).

Os dados contidos na Tabela 3 evidenciam que existe correlação positiva e significativa entre as variáveis número de ovos, número de insetos emergidos e número de sementes danificadas. Quanto ao dia de exposição das amostras à praga, embora 0 número de ovos tenha-se correlacionado negativamente, tal fonte de variação não influiu no número de insetos emergidos, nem sobre o número de sementes danificadas.
TABELA 3. Coeficientes de correlação de Pearson para probabilidade $>|\mathbf{R}|$, pressupondo Ho: $R h o=0, N=1224$, entre as variáveis dia de exposição, número de ovos, número de insetos emergidos e número de sementes danificadas.

\begin{tabular}{lccc}
\hline Variáveis & $\begin{array}{c}\text { Ovos } \\
\left(\log \mathrm{n}^{\circ}\right)\end{array}$ & $\begin{array}{c}\text { Insetos } \\
\text { emergidos } \\
\left(\log \mathrm{n}^{\circ}\right)\end{array}$ & $\begin{array}{c}\text { Sementes } \\
\text { danificadas } \\
\left(\log \mathrm{n}^{\circ}\right)\end{array}$ \\
\hline Dia de exposição & $-0,0941^{* *}$ & $-0,0119^{\mathrm{ns}}$ & $0,0167^{\mathrm{ns}}$ \\
$\mathrm{p}>|\mathrm{R}|$ & 0,0010 & 0,6753 & 0,5578 \\
Ovos & & $0,8859^{* *}$ & $0,6854^{* *}$ \\
$\quad \mathrm{p}>|\mathrm{R}|$ & 0,0001 & 0,0001 \\
Insetos emergidos & & & $0,7819^{* *}$ \\
$\mathrm{p}>|\mathrm{R}|$ & & 0,0001 \\
\hline **Significativo a $1^{\%}$ de probabilidade. & &
\end{tabular}




\section{CONCLUSÕES}

1. Os genótipos avaliados apresentam variabilidade quanto à preferência para postura, à antibiose, no que se refere ao número de ovos eclodidos, e ao número de sementes danificadas.

2. As linhas EVx 37-15E e EVx 37-2E são as mais resistentes ao ataque do caruncho.

3. As variáveis número de ovos, número de insetos emergidos e número de sementes danificadas são positivas e significativamente correlacionadas entre si.

4. As linhagens mais resistentes descendem de genitores resistentes.

\section{REFERÊNCIAS}

ARAÚJO, J.P.P. de; FREIRE FILHO, F.R.; SANTOS, J.H.R. dos. Melhoramento do caupi para resistência ao caruncho e ao manhoso. In: ARAUJO, J.P.P. de; WATT, E.E. (Orgs.). O caupi no Brasil. Brasília : Embrapa/IITA, 1988. p.301-322.

BASTOS, J.A.M. Avaliação dos prejuízos causados pelo gorgulho, Callosobruchus maculatus, em amostras de feijão-de-corda, Vigna sinensis, colhidos em Fortaleza, Ceará. Pesquisa Agropecuária Brasileira, Brasília, v.8, n.7, p.131-132, jul. 1973.

CHAVES, J.W.M.; VENDRAMIM, J.D. Não-preferência para oviposição e desenvolvimento de Callosobruchus maculatus (Fabr.) (Coleoptera: Bruchidae) em cultivares de caupi. Anais da Sociedade Entomológica do Brasil, Londrina, v.24, n.2, p.239-245, 1995.

FATUNLA, T.; BADARU, K. Resistance of cowpea pods to Callosobruchus maculatus Fabr. Journal of Agricultural Science, Cambridge, Grã-Bretanha, v.100, p.205-209, 1983

OLIVEIRA, F.J. de; SANTOS, J.H.R. dos; ALVES, J.F.; PAIVA, J.B.; ASSUNÇÃO, M.V. Perdas de peso em sementes de cultivares de caupi, atacadas pelo caruncho. Pesquisa Agropecuária Brasileira, Brasília, v.19, n.1, p.47-52, jan. 1984.

PESSOA, G.P.; BARROS, R.; OLIVEIRA, J.V. de. Avaliação da resistência de cultivares de caupi, Vigna unguiculata (L.) Walp., a Callosobruchus maculatus (Fabr.) em confinamento em laboratório. Anais da Sociedade Entomológica do Brasil, Londrina, v.22, n.2, p.259-266, 1993.

QUINDERÉ, M.A.W.; BARRETO, P.D. Suscetibilidade do caupi ao Callosobruchus maculatus (F.1775): estudos preliminares. Fortaleza : EPACE, 1982. 4p. (EPACE. Comunicado técnico, 13).

SANTOS, J.H.R. dos. Aspectos da biologia do Callosobruchus maculatus (Fabr., 1792) (Col., Bruchidae) sobre sementes de Vigna sinensis Endl. Piracicaba : ESALQ, 1971. 87p. Dissertação de Mestrado.

SANTOS, J.H.R. dos. Aspectos da resistência de cultivares de Vigna sinensis (L.) Savi ao ataque do Callosobruchus maculatus (F., 1775) (Col., Bruchidae), mantidos no Estado do Ceará-Brasil. Piracicaba : ESALQ, 1976. 194p. Tese de Doutorado.

SANTOS, J.H.R. dos; ALVES, J.F.; OLIVEIRA, F.J. de Perda de peso em sementes de Vigna sinensis (L.) Savi, decorrente do ataque de Callosobruchus maculatus (F., 1775) (Col., Bruchidae): primeira aproximação. Ciência Agronômica, Fortaleza, v.8, n.1/2, p.51-56, 1978.

SANTOS, J.H.R. dos; VIEIRA, F.V. Ataque do Callosobruchus maculatus (F.) a Vigna sinensis Endl. I. Influência sobre o poder germinativo de semente da cv. Seridó. Ciência Agronômica, Fortaleza, v.1, n.2, p.71-74, 1971.

SINGH, B.B.; SINGH, S.R.; ADJADI, O. Bruchid resistance in cowpea. Crop Science, Madison, v.25, n. 5, p. $736-739,1985$. 